Ciência Florestal, Santa Maria, v. 21, n. 1, p. 53-62, jan.-mar., 2011

\title{
PRODUÇ̃̃O DE BIOMASSA NO CORTE RASO EM PLANTIO DE Araucaria angustifolia (Bertol.) Kuntze DE 27 ANOS DE IDADE EM QUEDAS DO IGUAÇU, PR
}

\author{
BIOMASS PRODUCTION AFTER CLEAR CUTTING IN A 27-YEAR-OLD STAND \\ OF Araucaria angustifolia (Bertol.) Kuntze IN QUEDAS DO IGUAÇU, PR
}

\author{
Mauro Valdir Schumacher ${ }^{1}$ Rudi Witschoreck ${ }^{2}$ Francine Neves Calil $^{3}$
}

Vicente Guilheme Lopes ${ }^{2}$ Márcio Viera ${ }^{2}$

\begin{abstract}
RESUMO
Este trabalho, realizado em um povoamento de Araucaria angustifolia de 27 anos de idade no município de Quedas do Iguaçu (PR), teve como objetivos: estimar a biomassa nos diferentes componentes das árvores, no sub-bosque e na serapilheira acumulada sobre o solo. A biomassa foi estimada por meio do ajuste de equações de regressão, com coleta de 21 árvores distribuídas em sete classes diamétricas, serapilheira e o sub-bosque com base em unidades amostrais de área conhecida. A biomassa total do povoamento foi de 241,92 $\mathrm{Mg} \mathrm{ha}^{-1}$, sendo 82,2\% de Araucaria angustifolia, 9,6\% de sub-bosque e 8,2\% de serapilheira. Considerando apenas a biomassa de Araucaria angustifolia, 198,62 $\mathrm{Mg} \mathrm{ha}^{-1}$, madeira do tronco $(51,5 \%)$, casca do tronco $(14,7 \%)$, raiz $(13,0 \%)$, galhos vivos $(11,8 \%)$, grimpas (ramos aciculados) $(8,6 \%)$ e galhos mortos $(0,5 \%)$. A produção relativa de biomassa no componente madeira do tronco pode ser menor, quando comparado com outras espécies florestais, sobretudo por causa da elevada alocação no componente casca do tronco.
\end{abstract}

Palavras-chave: sustentabilidade; colheita florestal; sub-bosque; serapilheira.

\begin{abstract}
This study, conducted in a 27-year-old Araucaria angustifolia stand in the municipality of Quedas do Iguaçu (PR) county, aimed to estimate tree components, understory and accumulated litter biomasses. Biomass was estimated through regression equation adjustments, with 21 trees felled, distributed among 7 diametric classes, and with litter and understory based on known sample unit areas. Total stand biomass was 241.92 $\mathrm{Mg} \mathrm{ha}^{-1}$, with $82.2 \%$ composed of Araucaria angustifolia, 9.6\% composed of understory and 8.2\% composed by litter. Considering only Araucaria angustifolia, the biomass was of $198.62 \mathrm{Mg} \mathrm{ha}^{-1}$, composed of wood $(51.5 \%)$, bark $(14.7 \%)$, roots $(13.0 \%)$, lives branches $(11.8 \%)$, aciculated branches $(6.6 \%)$ and dead branches $(0.5 \%)$. Relative biomass production for the wood component may be low, when compared to other forest species, mainly due to the high allocation in the bark component.
\end{abstract}

Keywords: sustainability; forest harvesting; understory; litter.

1. Engenheiro Florestal, Dr., Professor Associado do Departamento de Ciências Florestais, Universidade Federal de Santa Maria, Av. Roraima 1000, CEP 97105-900, Santa Maria (RS). Bolsista do CNPq. schumacher@pq.cnpq.br

2. Engenheiro Florestal, Doutorando do Programa de Pós-Graduação em Engenharia Florestal, Universidade Federal de Santa Maria, Av. Roraima 1000, CEP 97105-900, Santa Maria (RS).rwitschoreck@yahoo.com.br

3. Engenheira Florestal, Dra . , Professora da Escola de Agronomia e Engenharia de Alimentos da Universidade Federal de Goiás, Rodovia Goiânia, Nova Veneza, km 0, Campus Samambaia, CEP 74690-900, Goiânia (GO). francine.calil@terra.com.br

Recebido para publicação em 14/08/2009 e aceito em 22/06/2010. 


\section{INTRODUÇÃO}

No Brasil, a área de distribuição natural da Araucaria angustifolia compreende os estados do Rio Grande do Sul, Santa Catarina e Paraná onde eram observados agrupamentos densos, sobretudo na parte leste e central do planalto sul-brasileiro. Também como ilhas esparsas no sul e nordeste do estado de São Paulo e mais raramente em algumas partes de Minas Gerais e Rio de Janeiro (HUECK, 1972, apud MACHADO e SIQUEIRA, 1980).

A Araucaria angustifolia ocorre naturalmente em zonas de clima mesotérmico dentro doesquema tipo $\mathrm{C}(\mathrm{Cfae} \mathrm{Cfb})$ seguindo a classificação de Köppen (MACHADO e SIQUEIRA, 1980), em altitude de 500 a $2.300 \mathrm{~m}$, mas preferencialmente de 500 a 1.500 m (CARVALHO, 1994).

A instalação de indústrias madeireiras juntamente com a queima indiscriminada para a formação de terras agricultáveis ocasionou a extinção em muitos lugares e a grande redução em outros, das outrora extensas reservas que, em razão de sua magnitude pareciam inacabáveis, de modo que hoje, matas primárias de Araucaria angustifolia são raramente encontradas em maciços extensos (MACHADO e SIQUEIRA, 1980).

A falta de estimulo à silvicultura de espécies nativas, além de aspectos legais, decorre da ausência de informações da autoecologia dessas espécies. A Araucaria angustifolia, por exemplo, que constituiu, durante mais de um século, a maior fonte de exportação de madeira de toda a América Latina, seja em virtude de sua ocorrência, constituindo, por vezes, agrupamentos gregários muito densos, ou por sua valiosa madeira, representa a mais importante espécie florestal, não só para o sul do Brasil como para toda a América Latina (REITZ et al., 1988).

$\mathrm{O}$ conhecimento do potencial de produtividade, melhor caracterizado pelo acúmulo de biomassa nos diferentes componentes das árvores, ao longo do ciclo produtivo e nas diferentes condições de desenvolvimento, é fundamental para a definição do manejo mais adequado.

Quanto à sustentabilidade ou manutenção da capacidade produtiva dos sítios florestais, informações sobre a partição da biomassa e dos nutrientes deveriam ser consideradas na definição de práticas como: idade e intensidade de colheita, manejo dos resíduos florestais, adubação de reposição, preparo de solo, entre outras.

$\mathrm{O}$ acúmulo de nutrientes, nos diversos componentes das árvores, apresenta grande variação ao longo das fases de desenvolvimento dos plantios florestais, por causa das diferenças de alocação de biomassa e concentração dos nutrientes (LANDSBERG, 1986; PALLARDY, 2008).

Turner e Lambert (1983) estudaram a ciclagem de nutrientes em um povoamento de Eucalyptus grandis e sugeriram que a colheita florestal não deve ser realizada antes dos 15 anos de idade, em consequência da elevada concentração de nutrientes no alburno.

Os ecossistemas florestais, naturais ou implantados, são sistemas abertos sujeitos a uma série de entradas e saídas de compostos inorgânicos e elementos químicos, muitos deles reconhecidamente essenciais para o desenvolvimento vegetal (ODUM, 1988; EPSTEIN e BLOOM, 2006; PALLARDY, 2008).

As entradas de nutrientes podem ocorrer através das deposições atmosféricas, secas e úmidas, intemperismo geológico, fixação biológica de nitrogênio e fertilização, enquanto as saídas incluem a volatilização pelas queimadas ou pela desnitrificação, lixiviação e erosão hídrica, assim como, pela colheita da biomassa (PRITCHETT, 1990).

Uma das principais vias de exportação de nutrientes, se não a mais importante, sobretudo em povoamentos florestais, é a colheita da biomassa (PRINCHETT, 1990; LANDSBERG, 1986). O entendimento da ciclagem de nutrientes nos ecossistemas florestais é um pré-requisito essencial para a compreensão e a predição dos efeitos da nutrição no crescimento da floresta (LANDSBERG, 1986).

Portanto, o presente trabalho teve como objetivo estimar a biomassa no corte raso de um povoamento de Araucaria angustifolia com 27 anos de idade, fracionada nos seguintes compartimentos: árvores de Araucaria angustifolia (grimpas (ramos finos com acícula), galhos vivos, galhos secos, casca do tronco, madeira do tronco e raiz); vegetação de sub-bosque (biomassa acima e abaixo do solo) e serapilheira acumulada.

\section{MATERIAL E MÉTODOS}

\section{Localização e caracterização da área do estudo}

O estudo foi realizado em um povoamento de Araucaria angustifolia, com 27 anos de idade, pertencente à empresa Araupel S.A., no município de Quedas do Iguaçu no estado do Paraná, localizado 
na latitude $25^{\circ} 26^{\prime} 38^{\prime \prime}$ sul e longitude $53^{\circ} 01^{\prime} 21^{\prime \prime}$ oeste.

O clima da região é o Cfa, subtropical mesotérmico, segundo a classificação de Köppen, com precipitação média anual de $1.900 \mathrm{~mm}$. A temperatura média anual é de $19,5^{\circ} \mathrm{C}$, sendo $38,1^{\circ} \mathrm{C}$ a máxima absoluta e $-5,5^{\circ} \mathrm{C}$ a mínima absoluta (IAPAR, 2010).

O solo é de baixa fertilidade como pode ser verificado nos valores médios de 15 amostras compostas coletadas na área do estudo, na Tabela 1, em que se destaca o elevado acúmulo de matéria orgânica, alta acidez e alumínio extraível e baixa saturação de bases.

\section{Amostragem e determinação da biomassa}

Com o objetivo principal de definir a amplitude diamétrica do povoamento, foram demarcadas cinco parcelas de $20 \mathrm{~m} \mathrm{x} 30 \mathrm{~m}$ onde foram medidos todos os DAPs (diâmetro com casca à altura do peito, 1,30 $\mathrm{m}$ de altura) com fita diamétrica e todas as alturas com hipsômetro da marca Suunto.

Tomando por base as informações do inventário florestal, a amplitude diamétrica foi dividida em sete classes de diâmetro, em cm: $(20,0-23,9 ; 24,0-27,9 ; 28,0-31,9 ; 32,0-35,9 ; 36,0-$ $39,9 ; 40,0-43,9$ e $\geq 44,0)$, com amostragem de três árvores distribuídas em cada classe, totalizando 21 árvores.

As árvores selecionadas foram seccionadas ao nível do solo e fracionadas nos seguintes componentes: grimpas (ramos finos com acícula), galhos vivos, galhos secos, casca do tronco, madeira do tronco e raiz. A biomassa de cada componente foi determinada por pesagem com balança de gancho.

Concomitantemente a amostragem de biomassa, foi feita uma cubagem rigorosa, segundo a metodologia de Smalian, com medições, com e sem casca, nas seguintes alturas ao longo do tronco: 0,$1 ; 0,3 ; 1,3 ; 2,3 ; 3,3 \mathrm{~m}$; e assim, sucessivamente, a intervalos de 1 metro, até o ápice da árvore.

O sistema radicular das árvores foi extraído por escavação manual e retroescavadeira, sendo posteriormente pesado após lavagem para retirada do solo aderido.

Para estimar a vegetação de sub-bosque, no centro de cada parcela do inventário florestal, foi avaliada uma parcela de $4 \mathrm{~m} \mathrm{x} 3 \mathrm{~m}$ onde toda a biomassa acima do solo foi coletada e pesada. Já para a avaliação da biomassa de raiz, em quatro parcelas, foi escavado um monólito de solo de $3 \mathrm{~m} \mathrm{x}$ $2 \mathrm{~m}$ e $0,6 \mathrm{~m}$ de profundidade. A biomassa acima do solo foi separada em diferentes frações para a coleta de amostras representativas. Uma fração herbácea (basicamente folhas e cipós sem consistência lenhosa) e cinco frações de troncos e galhos de acordo com o diâmetro: $<0,5 \mathrm{~cm} ; 0,6-3,5 \mathrm{~cm} ; 3,6-$ $6,5 \mathrm{~cm} ; 6,6-9,5 \mathrm{~cm}$ e $>9,5 \mathrm{~cm}$. O solo coletado foi passado por uma peneira e as raízes lavadas para retirar o solo aderido. As raízes de Araucaria angustifolia não foram consideradas na coleta de sub-bosque, as quais foram facilmente separadas das demais, com base na coloração e presença de micorizas.

Da serapilheira acumulada sobre o solo, foram coletadas nove amostras com moldura de ferro de $25 \mathrm{~cm} \mathrm{x} 25 \mathrm{~cm} \mathrm{em}$ cada uma das parcelas do inventário, perfazendo 45 amostras.

Para determinação do teor de umidade, foi coletada e pesada uma subamostra representativa de cada componente das árvores-amostra de Araucaria angustifolia e diferentes frações da biomassa de sub-bosque.

Todas as amostras de biomassa foram pesadas no campo com balança de precisão e acondicionadas em sacos de papel. No Laboratório de Ecologia Florestal do Departamento de Ciências Florestais/UFSM, as amostras foram secas a $70^{\circ} \mathrm{C}$, em estufa de circulação e renovação de ar, até a estabilização do peso e novamente pesadas para determinação da massa seca.

TABELA 1: Caracterização do solo da área do estudo, na profundidade de 0-20 cm.

TABLE 1: $\quad$ Soil characterization in the studied area, at a depth from 0 to $20 \mathrm{~cm}$.

\begin{tabular}{|c|c|c|c|c|c|c|c|c|c|c|c|}
\hline Argila & M.O. & \multirow{2}{*}{$\begin{array}{c}\mathrm{pH} \\
\left(\mathrm{H}_{2} \mathrm{O}\right)\end{array}$} & $\mathrm{P}$ & $\mathrm{K}$ & $\mathrm{Ca}$ & $\mathrm{Mg}$ & $\mathrm{Al}$ & $\mathrm{CTC}_{\text {efet }}$ & $\mathrm{CTC}_{\mathrm{pH} 7}$ & V & $\mathrm{m}$ \\
\hline \multicolumn{2}{|c|}{$(\%)$} & & \multicolumn{2}{|c|}{$\mathrm{mg} \mathrm{dm}{ }^{-3}$} & \multicolumn{5}{|c|}{$\mathrm{cmol}_{\mathrm{c}} \mathrm{dm}^{-3}$} & \multicolumn{2}{|c|}{$(\%)$} \\
\hline 60,3 & 5,1 & 4,1 & 1,4 & 36,0 & 1,3 & 0,3 & 2,9 & 4,7 & 14,0 & 12,8 & 61,6 \\
\hline
\end{tabular}

Em que: Extração do solo: P e K, solução Mehlich - 1; Ca e Mg, por solução de $\mathrm{KCl}(1$ mol L-1). 
A determinação da biomassa de Araucaria angustifolia e do sub-bosque foi realizada indiretamente por meio do teor de umidade. A serapilheira, em função da pequena quantidade de biomassa das unidades amostrais, a determinação da biomassa foi feita diretamente mediante secagem e pesagem de cada amostra.

\section{Estimativa de biomassa}

Após a determinação da biomassa seca, foram ajustadas equações de regressão, tendo como variáveis dependentes a biomassa de cada componente (grimpas, galhos vivos, galhos secos, casca do tronco, madeira do tronco e raiz) ou compartimento (biomassa acima do solo e biomassa total), por árvore, em $\mathrm{kg}$, e as seguintes variáveis independentes: dap (diâmetro com casca à altura do peito), h (altura total), bem como transformações aritméticas e logarítmicas (logaritmo natural) das variáveis dependentes e independentes.

A modelagem das equações de regressão seguiu o procedimento "forword" (proc stepwise e opção forward) do pacote SAS (1993), com verificação da normalidade da distribuição dos resíduos pelo teste de Shapiro-Wilk, homogeneidade da variância pelo teste de White e independência dos resíduos pela estatística de Durbin-Watson (SCHNEIDER et al., 2009).

As estatísticas utilizadas para verificar a qualidade do ajuste das equações de regressão foram: o coeficiente de determinação $\left(\mathrm{R}^{2}\right)$, erropadrão da estimativa absoluto (Syx), erro-padrão da estimativa relativo (Syx\%), além da análise da distribuição dos resíduos.

A estimativa da biomassa de Araucaria angustifolia por hectare foi realizada com aplicação das equações de regressão, selecionadas para cada componente, aos dados do inventário florestal. $\mathrm{O}$ valor médio obtido para cada componente da biomassa foi extrapolado com base na área da parcela do inventário.

Da mesma forma, a biomassa de sub-bosque e serapilheira foram estimadas em função da área e da biomassa média das unidades amostrais.

\section{RESULTADOS E DISCUSSÃO}

A Tabela 2 apresenta medidas descritivas para algumas variáveis dendrométricas, para a biomassa individual das árvores-amostra de
Araucaria angustifolia (kg/árvore) e para a biomassa das unidades amostrais de sub-bosque e serapilheira.

Considerando uma população de 376 árvores por hectare, ponderando em função da distribuição diamétrica, o povoamento de Araucaria angustifolia, aos 27 anos de idade, possui um volume de tronco, com e sem casca, respectivamente, de $288,4877 \mathrm{~m}^{3} \mathrm{ha}^{-1}$ e 232,6205 $\mathrm{m}^{3} \mathrm{ha}^{-1}$.

Apesar de um povoamento equiâneo, merece destaque a grande amplitude de variação, sobretudo, dos valores de biomassa dos diferentes componentes da biomassa das árvores de Araucaria angustifolia (Tabela 2).

No caso da serapilheira, apesar de ter sido coletado um número elevado de amostras, o coeficiente de variação foi alto. É provável que a utilização de unidades amostrais maiores que 25 $\mathrm{cm} \times 25 \mathrm{~cm}\left(625 \mathrm{~cm}^{2}\right)$ possa melhorar a estimativa da biomassa de serapilheira.

Utilizando unidades amostrais do mesmo tamanho $(25 \mathrm{~cm} \times 25 \mathrm{~cm})$, em plantios de Pinus taeda, respectivamente, com idades de 18 e 17 anos de idade, Schumacher et al., (2002) e Witschoreck (2008) estimaram coeficientes de variação $(\mathrm{CV})$ de $26,4 \%$ ( $n=24$ amostras) e $35,2 \%(n=54$ amostras $)$, para a serapilheira acumulada.

$\mathrm{Na}$ Tabela 3, são apresentadas as equações de regressão utilizadas para estimar a biomassa dos diferentes componentes das árvores de Araucaria angustifolia. É possível observar que são equações altamente significativas, estatisticamente, e com alto poder preditivo como se depreende pelos altos valores para os coeficientes de determinação e os baixos erros-padrão de estimativa.

Os modelos logarítmicos mostraramse mais eficientes para descrever a relação entre as variáveis dendrométricas e a biomassa dos diferentes componentes das árvores (Tabela 3). A grande aplicação de equações de regressão com transformação logarítmica de variáveis pode estar associada a problemas de heterogeneidade de variância dos dados de biomassa (Koehler et al. 2002; Schneider et al. 2009). Até mesmo as variáveis independentes selecionadas (Tabela 3) no processo de modelagem sugerem uma espécie de ponderação dos dados, o que, às vezes, contribui para a estabilidade da variância (Schneider et al. 2009).

A desrama natural que ocorre em povoamentos adultos, por causa do alto- 
sombreamento, provoca grande variabilidade na biomassa dos galhos secos, o que dificulta sua estimativa por equação de regressão. Como pode ser verificado na Tabela 3 , a estimativa desse componente, geralmente, está associada a uma maior margem de erro (SANQUETTA et al., 2003;

TABELA 2: Estatísticas descritivas de variáveis dendrométricas, biomassa individual das árvores-amostra de Araucaria angustifolia e biomassa das unidades amostrais de sub-bosque e serapilheira.

TABLE 2: Descriptive statistics of dendrometric variables, biomass of each Araucaria angustifolia tree and biomass from sampling units of understory and litter.

\begin{tabular}{|c|c|c|c|c|c|c|}
\hline Variável & Unidade & $\mathrm{N}$ & Média & CV $(\%)$ & Mínimo & Máximo \\
\hline Dap (cm) & $\mathrm{cm}$ & 21 & 34,0 & 25,1 & 22,30 & 49,30 \\
\hline Altura (m) & $\mathrm{m}$ & 21 & 19,8 & 9,2 & 16,40 & 23,00 \\
\hline Volume do tronco com casca & \multirow{2}{*}{$\mathrm{m}^{3}$} & 21 & 1,0720 & 50,9 & 0,4026 & 1,9695 \\
\hline Volume do tronco sem casca & & 21 & 0,8636 & 51,1 & 0,3193 & 1,6265 \\
\hline Grimpa & \multirow{7}{*}{$\mathrm{kg}$} & 21 & 68,37 & 68,6 & 13,04 & 153,40 \\
\hline Galho vivo & & 21 & 95,92 & 67,2 & 22,55 & 240,48 \\
\hline Galho seco & & 21 & 5,12 & 87,3 & 0,57 & 18,18 \\
\hline Casca do tronco & & 21 & 100,62 & 53,4 & 30,82 & 232,32 \\
\hline Madeira do tronco & & 21 & 402,91 & 54,7 & 135,12 & 820,02 \\
\hline Raiz & & 21 & 105,56 & 67,9 & 27,06 & 257,06 \\
\hline Árvore inteira & & 21 & 778,60 & 57,8 & 242,51 & 1549,90 \\
\hline \multirow{2}{*}{ Sub-bosque } & \multirow{2}{*}{$\mathrm{kg}$} & 5 & 20,15 & 23,7 & 13,80 & 26,62 \\
\hline & & 4 & 3,84 & 24,4 & 2,67 & 4,95 \\
\hline Serapilheira & $\mathrm{g}$ & 45 & 123,55 & 35,0 & 65,30 & 235,30 \\
\hline
\end{tabular}

Em que: $\mathrm{N}$ = número de observações; $\mathrm{CV}(\%)$ = coeficiente de variação percentual; dap = diâmetro à altura do peito.

TABELA 3: Equações de regressão para estimar a biomassa nos diferentes componentes das árvores de Araucaria angustifolia com 27 anos de idade.

TABLE 3: Regression equations for estimating biomass in different components of 27-year-old Araucaria angustifolia trees.

\begin{tabular}{|c|c|c|c|c|c|c|}
\hline & Componente & \multicolumn{5}{|c|}{ Equação } \\
\hline 1 & Grimpas & & & \multicolumn{3}{|c|}{$\ln y=b_{0}+b_{1} \cdot d^{-1}$} \\
\hline 2 & \multicolumn{3}{|c|}{ Galhos vivos } & \multicolumn{3}{|c|}{$\ln y=b_{0}+b_{1} . \operatorname{lnd}$} \\
\hline 3 & \multicolumn{3}{|c|}{ Galhos secos } & \multicolumn{3}{|c|}{$\ln y=b_{0}+b_{1} \cdot \ln \left(d \cdot h^{2}\right)$} \\
\hline 4 & \multicolumn{3}{|c|}{ Casca do tronco } & \multicolumn{3}{|c|}{$\ln y=b_{0}+b_{1} \cdot d^{-1}$} \\
\hline 5 & \multicolumn{3}{|c|}{ Madeira do tronco } & \multicolumn{3}{|c|}{$\ln y=b_{0}+b_{1} \cdot \ln \left(d^{3} \cdot h\right)$} \\
\hline 6 & \multicolumn{3}{|c|}{ Raiz } & \multicolumn{3}{|c|}{$\ln y=b_{0}+b_{1} . \operatorname{lnd}$} \\
\hline 7 & \multicolumn{3}{|c|}{ Biomassa acima do solo (total - raiz) } & \multirow{2}{*}{\multicolumn{3}{|c|}{$\begin{array}{l}\ln y=b_{0}+b_{1} . \ln d \\
\ln y=b_{0}+b_{1} . \ln d\end{array}$}} \\
\hline 8 & \multicolumn{3}{|c|}{ Biomassa total } & & & \\
\hline \multicolumn{7}{|c|}{ Coeficientes e estatísticas de ajuste } \\
\hline & $\mathrm{b}_{0}$ & $\mathrm{~b}_{1}$ & Prob. $>$ F & $\mathrm{R}^{2}$ & Syx & Syx $(\%)$ \\
\hline 1 & 7,02021 & $-98,28230$ & 0,0001 & 0,931 & 0,2198 & 5,57 \\
\hline 2 & $-5,29730$ & 2,75159 & 0,0001 & 0,899 & 0,2414 & 5,58 \\
\hline 3 & $-12,33995$ & 1,44564 & 0,0001 & 0,566 & 0,5427 & 41,00 \\
\hline 4 & 6,67244 & - 70,67524 & 0,0001 & 0,948 & 0,1355 & 3,04 \\
\hline 5 & $-3,52285$ & 0,69518 & 0,0001 & 0,960 & 0,1229 & 2,10 \\
\hline 6 & $-5,38290$ & 2,80408 & 0,0001 & 0,958 & 0,1532 & 3,41 \\
\hline 7 & $-2,09238$ & 2,41141 & 0,0001 & 0,965 & 0,1208 & 1,91 \\
\hline 8 & $-2,12394$ & 2,46033 & 0,0001 & 0,971 & 0,1124 & 1,73 \\
\hline
\end{tabular}

Em que: $\ln =$ logaritmo natural; $\mathrm{b}_{0}, \mathrm{~b}_{1}=$ coeficientes; $\mathrm{y}=$ biomassa em $\mathrm{kg}$ do respectivo componente; $\mathrm{d}=$ dap (diâmetro à altura do peito); $\mathrm{h}=$ altura total; Prob.> $\mathrm{F}=$ nível de significância para a estatística $\mathrm{F} ; \mathrm{R}^{2}=$ coeficiente de determinação; Syx = erro-padrão de estimativa absoluto; Syx $(\%)=$ erro-padrão de estimativa relativo. 
SCHUMACHER et al., 2003; SCHUMACHER e WITSCHORECK, 2008).

Schumacher et al., (2003) e Schumacher e Witschoreck, (2008) calcularam um erro na estimativa da biomassa de galhos secos, respectivamente, para Eucalyptus spp. e Pinus taeda, de 38,9\% e 43,3\%. Enquanto Sanquetta et al. (2003), para biomassa verde, estimou um erro para galhos mortos, que variou de $83,41 \%$ a $100,66 \%$ para diferentes modelos de regressão, para árvores de Araucaria angustifolia de plantios entre 29 e 33 anos de idade. Porém, em razão da pequena biomassa relativa desse componente (Tabela 4 ), dificilmente o erro associado à sua estimativa compromete a determinação da biomassa total, tanto que alguns estudos o consideram junto com os galhos vivos.

$\mathrm{Na}$ Tabela 4, é possível verificar a distribuição absoluta e relativa da biomassa estimada nos diferentes compartimentos do povoamento florestal.

Considerando as árvores de Araucaria angustifolia, a sequência de acúmulo de biomassa nos diferentes componentes obedece à seguinte ordem: madeira do tronco $>$ casca do tronco $>$ raiz $>$ galhos vivos $>$ grimpas $>$ galhos secos.

Para a mesma espécie florestal, Watzlawick et al. (2003) quantificaram a biomassa em um povoamento de 30 anos de idade e densidade de 540 árvores por hectare, em General Carneiro no Paraná, e determinaram a mesma sequência de acúmulo de biomassa, ou seja: madeira do tronco $(42,50 \%)$, casca do tronco $(23,80 \%)$, raiz $(15,75 \%$, galhos vivos $(11,64 \%)$, grimpas $(7,64 \%)$ e galhos mortos $(1,18 \%)$.

Sanquetta et al. (2003) avaliaram as relações individuais de biomassa em plantações de Pinus taeda (considerando a biomassa acima e abaixo do solo de árvores de 14 a 32 anos de idade) e Araucaria angustifolia (considerando a biomassa acima do solo de árvores de 29 a 33 anos de idade), no sul do estado do Paraná, e determinaram a seguinte partição de biomassa verde, respectivamente: fuste, 59,00 e 54,22\%, casca, 9,75 e 17,74\%; galhos vivos 13,62 e 17,66\%; folhagem, 4,27 e 8,76\%; galhos mortos, 1,89 e $1,62 \%$

Krapfenbauer e Andrae (1983), ao estudar a alocação de biomassa em um povoamento de Araucaria angustifolia de 17 anos de idade e 1.220 plantas por hectare, no Rio Grande do Sul, determinaram a seguinte partição de biomassa: madeira do tronco $(50,5 \%)$, grimpas $(15,1 \%)$, casca do tronco $(14,2 \%)$, raiz $(12,4 \%)$ e galhos (7,8\%). Krapfenbauer e Andrae (1983) também estimaram a biomassa de serapilheira acumulada,

TABELA 4: Produção e partição de biomassa estimada para a serapilheira, sub-bosque e para os diferentes componentes das árvores de Araucaria angustifolia, com 27 anos de idade.

TABLE 4: Estimated biomass production and partition for litter, understory and different components of 27-year-old Araucaria angustifolia trees.

\begin{tabular}{|c|c|c|c|c|c|}
\hline \multirow{3}{*}{ Compartimento } & \multirow{3}{*}{ Componente } & \multirow{3}{*}{$\begin{array}{l}\text { Biomassa } \\
\left(\mathrm{Mg} \mathrm{ha}^{-1}\right)\end{array}$} & \multicolumn{3}{|c|}{ Biomassa relativa $(\%)$} \\
\hline & & & \multirow{2}{*}{ Total } & \multicolumn{2}{|c|}{ Arbórea } \\
\hline & & & & Total & Acima do solo \\
\hline \multirow{9}{*}{$\begin{array}{c}\text { Araucaria } \\
\text { angustifolia }\end{array}$} & Grimpas & 17,04 & 7,0 & 8,6 & 9,8 \\
\hline & Galhos vivos & 23,45 & 9,7 & 11,8 & 13,5 \\
\hline & Galhos secos & 0,94 & 0,4 & 0,5 & 0,5 \\
\hline & Copa (grimpa + galhos) & 41,43 & 17,1 & 20,8 & 23,9 \\
\hline & Casca do tronco total & 29,20 & 12,1 & 14,7 & 16,9 \\
\hline & Madeira do tronco total & 102,53 & 42,4 & 51,5 & 59,2 \\
\hline & Biomassa acima do solo & 173,16 & 71,6 & 87,0 & 100,0 \\
\hline & Raiz & 25,80 & 10,7 & 13,0 & \\
\hline & Biomassa arbórea total & 198,96 & 82,2 & 100,0 & \\
\hline \multirow{3}{*}{ Sub-bosque } & Acima do solo & 16,79 & 6,9 & & \\
\hline & Raiz & 6,40 & 2,6 & & \\
\hline & Total & 23,19 & 9,6 & & \\
\hline \multicolumn{2}{|c|}{ Serapilheira } & 19,77 & 8,2 & & \\
\hline \multicolumn{2}{|c|}{ Biomassa total } & 241,92 & 100,0 & & \\
\hline
\end{tabular}

Ci. Fl., v. 21, n. 1, jan.-mar., 2011 
que apresentava alguns resíduos de desbaste, e foi de $17,3 \mathrm{Mg} \mathrm{ha}^{-1}$. Enquanto, para a mesma espécie florestal e região do presente estudo, Schumacher et al. (2000) quantificaram, para um povoamento de 14 anos de idade e uma densidade de 1.600 árvores por hectare, $11,8 \mathrm{Mg} \mathrm{ha}^{-1}$.

A serapilheira acumulada resultada do equilíbrio entre as taxas de deposição e de decomposição dos resíduos. Nesse sentido, grande aporte de resíduos orgânicos pode ser compensado por altas taxas de decomposição (taxa de decaimento), o que está relacionado com a espécie florestal, idade, condições climáticas, qualidade do sítio, entre outros fatores (REIS e BARROS, 1990).

A taxa de decomposição microbiológica dos resíduos orgânicos depende das características químicas do material, no que concerne ao conteúdo de nutrientes, especialmente nitrogênio (relação $\mathrm{C} / \mathrm{N}$ ), presença de moléculas orgânicas como lignina e compostos fenólicos; atividade da microfauna do solo e das condições do ambiente, sobretudo temperatura e umidade (BINKLEY, 1986; WARING e SCHLESINGER, 1985).

Poggiani (1985) estudou, em duas áreas disjuntas de São Paulo, um povoamento de Eucalyptus saligna com 11 anos de idade e um de Pinus caribaea com 14 anos de idade onde verificou, respectivamente, uma deposição média de serapilheira, de 4,49 $\mathrm{Mg} \mathrm{ha}^{-1}$ ano $^{-1}$, com predomínio durante o verão; e 8,37 $\mathrm{Mg} \mathrm{ha}^{-1}$ ano $^{-1}$, concentrada nos períodos secos durante a primavera e o outono. No mesmo trabalho, a serapilheira acumulada sobre o solo foi estimada em 7,94 $\mathrm{Mg} \mathrm{ha}^{-1}$ no Eucalyptus saligna e 20,24 $\mathrm{Mg} \mathrm{ha}^{-1}$ no povoamento de Pinus caribaea.

$\mathrm{Na}$ região de Cambará do Sul (RS), Schumacher et al. (2007) estimaram uma deposição de serapilheira em povoamento de Pinus taeda, respectivamente, para o $5^{\circ}, 6^{\circ}$ e $7^{\circ}$ anos de idade, em Mg ha ${ }^{-1}$, de 3,99; 4,72 e 4,84. Enquanto em termos de serapilheira acumulada, na mesma região, Schumacher et al. (2002) obtiveram, para Pinus taeda, aos 18 anos de idade, uma biomassa de 16 $\mathrm{Mg} \mathrm{ha}^{-1}$.

Para Arancaria angustifolia, em povoamento de 17 anos de idade, plantado em espaçamento de 2 × $2 \mathrm{~m}$ (densidade inicial de 2500 árvores), Schumacher et al. (2004), em Pinhal Grande (RS), determinaram uma deposição de serapilheira de 6,96 $\mathrm{Mg} \mathrm{ha}^{-1}$, apresentando picos entre a primavera e o verão, associados com as maiores precipitações médias mensais.
$\mathrm{Na}$ Tabela 4, é possível verificar que o subbosque apresenta 23,19 $\mathrm{Mg} \mathrm{ha}^{-1}$ o que representa $9,6 \%$ da biomassa total do povoamento.

Waring e Schlesinger (1985) afirmam que, em consequência da alta concentração de nutrientes e a elevada proporção da folhagem em relação ao outros tecidos, o sub-bosque tem mais importância na ciclagem de nutrientes do que a sua biomassa sugere.

Costa (1990) salienta a importância da heterogeneidade da comunidade vegetal que, no caso do sub-bosque de plantios florestais, pode corroborar para a manutenção dos nutrientes no sistema solo-planta. Em função das diferentes exigências nutricionais, quanto maior a heterogeneidade da comunidade vegetal, melhor será o equilíbrio dos nutrientes no ecossistema.

Nesse contexto, além de outras funções, o sub-bosque atua como um compartimento de transição para os nutrientes, o que otimiza a absorção e retenção dos nutrientes (evitando sua saída da zona radicular) e disponibilizando os nutrientes, posteriormente, de forma lenta e gradual por meio da decomposição dos resíduos.

A idade do povoamento florestal é um dos fatores que mais influencia na distribuição de biomassa, estando relacionado com os diferentes estágios nutricionais das árvores (GONÇALVES et al., 2004).

Ao longo do desenvolvimento do povoamento florestal, ainda que ocorra um incremento absoluto, a biomassa relativa de madeira do tronco tende a aumentar em detrimento dos demais componentes (PEREIRA et al., 1984; WARING e SCHLESINGER, 1985; VALERI et al., 1988; SCHUMACHER et al., 2003; GONÇALVES et al., 2004; MARTIN et al., 2004). Portanto, quando comparada à alocação de biomassa de uma mesma espécie, em diferentes idades, é comum encontrarse maior acúmulo relativo nos componentes da copa nos povoamentos mais jovens.

$\mathrm{O}$ espaçamento entre plantas é outro fator que interfere na distribuição de biomassa nos diferentes componentes das árvores (LEITE et al., 1997; LELES et al. 1998; LADEIRA et al., 2001).

$\mathrm{Na}$ região deste estudo em um plantio de Araucaria angustifolia de 14 anos de idade, porém, sob uma densidade de 1.600 árvores por hectare aos 14 anos de idade, Schumacher et al. (2000) quantificaram uma partição de biomassa acima do solo muito semelhante a atual, mas com maior acúmulo na madeira, qual seja: madeira do tronco 
$64 \%$, casca do tronco $14 \%$, grimpa $11 \%$, galho vivo $8 \%$ e galho seco $3 \%$.

Baldwin Jr. et al. (2000), ao estudarem o efeito do espaçamento e dos desbastes em Pinus taeda, aos 38 anos de idade, verificaram que os povoamentos mais intensamente desbastados apresentaram alteração no padrão de distribuição de biomassa, em especial, alocando mais biomassa para galhos e folhas.

Leite et al. (1997) e Ladeira et al. (2001) avaliaram a produção de biomassa de eucalipto, sob diferentes espaçamentos, e verificaram que à medida que a densidade populacional aumenta, a biomassa total por unidade de área aumenta, ao passo que a produção total por indivíduo diminui, mas, com elevação da alocação de fotoassimilados no tronco.

Em razão da dificuldade de amostragem da biomassa abaixo do solo, geralmente o sistema radicular não é considerado, porém não é difícil verificar a sua importância em termos de biomassa relativa (Tabela 4 ).

Reis e Barros (1990) salientaram a importância do sistema radicular nos estudos que avaliam a demanda de nutrientes para o crescimento da planta, bem como quando o objetivo for analisar diferentes métodos de manejo sobre a produtividade das rotações subsequentes, especialmente no que se refere a espécies que regeneram por brotação.

Quanto à partição de biomassa, o sistema radicular e, consequentemente, a relação parte aérea/raiz, é especialmente afetado pelas condições de fertilidade do solo (REIS et al., 1985; REIS et al., 1987). Essa relação é de 6,7 para a Araucaria angustifolia, 2,6 para o sub-bosque.

Reis et al. (1985), em uma sequência de idade de Eucalyptus grandis, estimaram a biomassa relativa do sistema radicular, em duas áreas com diferentes níveis de produtividade, em 13,2 e 32,0\%, respectivamente, para a área de maior e de menor produtividade; enquanto a biomassa de raízes com diâmetro menor do que $3 \mathrm{~mm}$ era 2,6 vezes maior no sítio menos produtivo.

Do mesmo modo, Bizon (2005), quando estudou oito povoamentos de Pinus taeda, atribuiu aos diferentes níveis de produtividade, a diferença na alocação de biomassa para o sistema radicular, que variou de 16 para $22 \%$, em sítios de maior e de menor produtividade, respectivamente.

Com exceção de povoamentos extremamente jovens, a madeira do tronco é o componente das árvores que apresenta a maior biomassa relativa (REIS e BARROS, 1990).

$\mathrm{O}$ acúmulo de $51,5 \%$ da biomassa arbórea total na madeira do troco é bastante expressivo, no entanto, é possível encontrar valores maiores para outras espécies florestais (Tabela 4).

Considerando uma espécie mais "próxima" à Araucaria angustifolia, o Pinus taeda, Witschoreck (2008) estimou a madeira do tronco em $62,4 \%$ da biomassa total em um povoamento de 17 anos de idade e 581 árvores por hectare, em Cambará do Sul (RS). E analisando apenas a biomassa acima do solo, Schumacher e Witschoreck (2008) estimaram em $69,1 \%$ a biomassa de madeira do tronco aos 27 anos de idade, sob densidade de 243 árvores por hectare.

Desse modo, é possível afirmar que a Araucaria angustifolia é uma espécie que apresenta menor acúmulo relativo de biomassa na madeira do tronco em comparação ao Pinus taeda, o que em parte, está relacionado à maior alocação relativa na casca. Neste estudo, verificou-se um valor de 14,7\%. Valeri (1988), Bizon (2005), Witschoreck (2008) e Schumacher e Witschoreck (2008), estimaram a alocação na casca do tronco, em termos médios, duas vezes inferior, para Pinus taeda.

Os dados obtidos por Sanquetta et al. (2003), em povoamentos de Pinus taeda e Araucaria angustifolia, com idades entre 29 e 33 anos, 14 e 32 anos respectivamente, e Watzlawick et al. (2003) para Araucaria angustifolia aos 30 anos de idade; ambos em General Carneiro (PR), também corroboram esse padrão de alocação para as duas espécies.

\section{CONCLUSÕES}

Considerando os compartimentos Aracucaria angustifolia, sub-bosque e serapilheira acumulada, a partição de biomassa apresentou a seguinte distribuição: $82,2, \quad 9,6$ e $\quad 8,2 \%$ respectivamente.

O padrão de alocação de biomassa nos diferentes componentes das árvores de Araucaria angustifolia apresentou a seguinte sequência de acúmulo: madeira do tronco $(51,5 \%)$, casca do tronco $(14,7 \%)$, raiz $(13,0 \%)$, galhos vivos $(11,8 \%)$, grimpas $(8,6 \%)$ e galhos mortos $(0,5 \%)$.

As relações parte aérea/raiz da biomassa de Arancaria angustifolia e do sub-bosque foram estimadas em 6,7 e 2,6, o que corresponde a uma alocação acima e abaixo do solo, respectivamente, de $87 \%$ e $13 \%$ e $72,4 \%$ e $27,6 \%$.

Ci. Fl., v. 21, n. 1, jan.-mar., 2011 
A produção relativa de biomassa no componente madeira do tronco de Araucaria angustifolia pode ser menor, quando comparado com outras espécies florestais, especialmente por causa da elevada alocação no componente casca do tronco, o que pode ser o dobro, em termos relativos, dos valores estimados para algumas espécies, como Pinus taeda.

\section{AGRADECIMENTOS}

À empresa Araupel S.A. pela cessão da área para realização deste trabalho, bem como disponibilização de recursos humanos e materiais para coletas de campo.

\section{REFERÊNCIAS BIBLIOGRÁFICAS}

BALDWIN Jr. et al. The effects of spacing and thinning on stand and tree characteristics of 38 -year-old loblolly pine. Forest Ecology and Management, Amsterdam, v. 137, n. 1-3, p. 91102, 15 Oct. 2000.

BINKLEY, D. Forest nutrition management. New York: John Wiley \& Sons, 1986. 290 p.

BIZON, J. M. C. Avaliação da sustentabilidade nutricional de plantios de Pinus taeda $\mathrm{L}$. usando um balanço de entrada-saída de nutrientes. 2005. 95 f. Dissertação (Mestrado em Recursos Florestais)-Escola Superior de Agricultura Luiz de Queiroz, Piracicaba, 2005.

CARVALHO, P. E. R. Espécies florestais brasileiras: recomendações silviculturais, potencialidades e uso da madeira. Colombo: EMBRAPA-CNPF, 1994. 640 p.

COSTA, L. M. Manejo de solos em áreas florestais. In: BARROS, N. F.; NOVAIS, R. F. (eds.) Relação solo eucalipto. Viçosa: Ed. Folha de Viçosa, 1990. cap. 6, p. 237-302.

EPSTEIN, E.; BLOOM, A. J. Nutrição mineral de plantas. Londrina, Ed. Planta, 2006. 403 p.

GONÇALVES, J. L. M. et al. An evaluation of minimum and intensive soil preparation regarding fertility and tree nutrition. In: GONÇALVES, J. L. M. e BENEDETTI, V. (eds.). Forest nutrition and fertilization. Piracicaba: IPEF, 2004. cap. 2, p. 1364.

IAPAR - Instituto Agronômico do Paraná. Disponível em: < (http://www.iapar.br/modules/ conteudo/conteudo.php?conteudo $=677)>$ acessado em: 10 de maio de 2010.

KOEHLER, H. S.; WATZLAWICK, L. F.;
KIRCHNER, F. F. Fontes e níveis de erros nas estimativas do potencial de fixação de carbono. In: Sanquetta, C. R. et al. (eds.). As florestas e o carbono. Curitiba: UFPR, 2002. cap. 13, p. 251264.

KRAPFENBAUER, A.; ANDRAE, F. Inventário de um reflorestamento de araucária de 17 anos em Passo Fundo - RS: inventário da biomassa. In: ANDRAE, F.; KRAPFENBAUER, A. Pesquisa austríaco-brasileira (1973-1982). Santa Maria, 1983. p. 30-55.

LADEIRA, B. C. et al. Produção de biomassa de eucalipto sob três espaçamentos em uma seqüência de idade. Revista Árvore, Viçosa, v. 25, n. 1, p. 6978, jan./mar. 2001.

LANDSBERG, J. J. Physiological ecology of forest production. London: Academic Press, 1986. 198 p. LEITE, F. P. et al. Crescimento de Eucalyptus grandis em diferentes densidades populacionais. Revista Árvore, Viçosa, v. 21, n. 3, p. 313-321, jun./set. 1997.

LELES, P. S. S. et al. Relações hídricas e crescimento de árvores de Eucalyptus camaldulensis e Eucalyptus pellita sob diferentes espaçamentos na região de cerrado. Revista Árvore, Viçosa, v. 22, n. 1, p. 41-50, jan./mar. 1998.

MACHADO, S. A.; SIQUEIRA, J. D. P. Distribuição natural da Araucaria angustifolia (Bertol.) Kuntze In: PROBLEMAS FLORESTAIS DO GÊNERO ARAUCARIA, 1979, Curitiba. Anais... Curitiba: IUFRO, 1980. p. 4-9.

MARTIN, T. A.; JOKELA, E. J. Stand development and production dynamics of loblolly pine under a range of cultural treatments in north-central Florida USA. Forest Ecology and Management, Amsterdam, v. 192, n. 1, p. 39-58, 19 Apr. 2004.

ODUM, E. P. Ecologia. Rio de Janeiro: Guanabara Koogan, 1988. 434 p.

PALLARDY, S. Physiology of woody plants. San Diego: Academic Press, 2008. 454 p.

PEREIRA, A. R. et al. Concentração e distribuição de nutrientes em Eucalyptus grandis em função da idade, cultivado na região do cerrado. Brasil Florestal, Brasília, n. 59, p. 27 - 37, jun./set. 1984. POGGIANI, F. Nutrient cycling in Eucalyptus and Pinus plantations ecosystems. Silvicultural implications. IPEF, Piracicaba, n. 31, p. 33-40, dez. 1985.

PRITCHETT, W. L. Suelos forestales: propriedade, conservación y mejoramento. México: Limusa Noriega, 1990. $634 \mathrm{p}$.

REIS, M. G. F.; BARROS, N. F. Ciclagem de 
nutrientes em plantios de eucalipto. In: BARROS, N. F.; NOVAIS, R. F. (eds). Relação solo eucalipto. Viçosa: Ed. Folha de Viçosa, 1990. cap. 7, p. 265302.

REIS, M. G. F.; BARROS, N. F.; KIMMINS, J. P. Acúmulo de nutrientes em uma seqüência de idade de Eucalyptus grandis W. Hill ex Maiden plantado no cerrado, em duas áreas com diferentes produtividades, em Minas Gerais. Revista Árvore, Viçosa, v. 11, n. 1, p. 1-15, jan./jun. 1987.

REIS, M. G. F. et al. Acúmulo de biomassa em uma seqüência de idade de Eucalyptus grandis plantado no cerrado em duas áreas com diferentes produtividades. Revista Árvore, Viçosa, v. 9, n. 2, p. 149-162, jul./dez. 1985.

REITZ, R.; KLEIN, R. M.; REIS, A. Projeto madeira do Rio Grande do Sul. Herbário Barbosa Rodrigues, Governo do Estado do Rio Grande do Sul, 1988. 525 p.

SANQUETTA, C. R. et al. Relações individuais de biomassa e conteúdo de carbono em plantações de Araucaria angustifolia e Pinus taeda no sul do estado do Paraná, Brasil. Revista Acadêmica, Curitiba, v. 1, n. 3, p. 33-40, jul./set. 2003.

SAS. Statistical analysis system: Programa de computador, ambiente VM. Cary, 1993. Versão 6.08 .

SCHUMACHER, M. V.; HOPPE, J. M.; BARBIERI, S. J. Quantificação da biomassa e do conteúdo de nutrientes no primeiro desbaste de uma floresta de Araucaria angustifolia (Bertol.) Kuntze na região de Quedas do Iguaçu - PR. Santa Maria: UFSM/ DCFL/CEPEF. 2000. 43 p. (Relatório Técnico).

SCHUMACHER, M. V.; COPETTI, L.; WITSCHORECK, R. Quantificação da biomassa e nutrientes em um povoamento de Pinus taeda aos 18 anos de idade na região de Cambará do Sul - RS. Santa Maria: UFSM/FATECIENS, Departamento de Ciências Florestais. 2002. 47 p. (Relatório Técnico).

SCHUMACHER, M. V. et al. Quantificação do carbono e dos nutrientes em florestas de eucalipto de diferentes idades. Santa Maria: UFSM/FATECIENS, Departamento de Ciências
Florestais, 2003. 112 p. (Relatório Técnico). SCHUMACHER, M. V. et al. Produção de serapilheira em uma floresta de Araucaria angustifolia (Bertol.) Kuntze no município de Pinhal Grande-RS. Revista Árvore, Viçosa, v. 28, n. 1, p. 29-37, jan./fev. 2004.

SCHUMACHER, M. V. et al. Devolução de serapilheira e nutrients em área de segunda rotação com Pinus taeda L. Santa Maria: UFSM/ FATECIENS, Departamento de Ciências Florestais, 2007. 27 p. (Relatório Técnico).

SCHUMACHER, M. V.; WITSCHORECK, R. Quantificação da biomassa e dos nutrientes no corte raso de um povoamento de Pinus taeda $\mathbf{L}$. em Cambará do Sul - RS. Santa Maria: UFSM/ FATECIENS, Departamento de Ciências Florestais. 2008. 37 p. (Relatório Técnico).

SCHNEIDER, P. R.; SCHNEIDER, P. S. P.; SOUZA, C. A. M. Análise de regressão aplicação à engenharia florestal. 2. ed. rev. e ampl. Santa Maria: FACOS, 2009. 294 p.

TURNER, J.; LAMBERT, M. J. Nutrient cycling within a 27-year-old Eucalyptus grandis plantation in New South Wales. Forest Ecology and Management. v. 6, n. 2, p. 155-168. July 1983.

VALERI, S. V. Exportação de biomassa e nutrientes de povoamentos de Pinus taeda $\mathbf{L}$. desbastados em diferentes idades. 1988. 164 f. Tese (Doutorado em Engenharia Florestal)Universidade Federal do Paraná, Curitiba, 1988.

WARING, R. H.; SCHLESINGER, W. H. Forest ecosystems: concepts and management. San Diego: Academic Press, 1985. 340 p.

WATZLAWICK, L. F. et al. Quantificação de biomassa total e carbono orgânico em povoamentos de Araucaria angustifolia (Bertol.) Kuntze no sul do estado do Paraná, Brasil. Revista Acadêmica, Curitiba, v. 1, n. 2, p. 63-68, abr./jun. 2003.

WITSCHORECK, R. Biomassa e nutrientes no corte raso de um povoamento de Pinus taeda $\mathbf{L}$. de 17 anos de idade no município de Cambará do Sul - RS. 2008. 80 f. Dissertação (Mestrado e Engenharia Florestal)-Universidade Federal de Santa Maria, Santa Maria, 2008. 